\title{
Knöcherne Verletzungen der Phalangen
}

\author{
Holger C. Erne, Hans-Günther Machens
}

\section{Zusammenfassung}

Knöcherne Verletzungen der Phalangen gehören aufgrund der Exponiertheit der Hände zu den häufigsten Verletzungen. Bei der Frakturversorgung, ob konservativ oder operativ, sollte immer die zu erhaltende Beweglichkeit im Blick behalten werden. Eine zu lange Ruhigstellung, ein überstehender K-Draht, die zu spät begonnene Handtherapie oder die Therapie durch handchirurgisch nicht geschulte Physiotherapeuten führen $\mathrm{zu}$ dauerhaft eingeschränktem Bewegungsausmaß und damit zur Einschränkung der Arbeitsfähigkeit und Lebensqualität.

\section{Bony Injuries of the Phalangeas}

Bony injuries of the phalangeas are frequent due to the exposure of the hand. With the treatment preserving the range of motion must be kept in the view. Too long immobilization, projecting k-wire, too late begun physiotherapy, poorly trained therapists are reasons which lead frequently to a constrained range of motion and thereby to reduced employability and quality + of life.

\section{Versorgungsprinzipien}

Knöcherne Verletzungen der Phalangen sollten möglichst innerhalb der 1 . Woche nach dem Trauma definitiv versorgt werden. Stabile, nicht dislozierte Phalanxfrakturen können konservativ behandelt werden. Es sollte dabei immer die Hauptgefahr der längeren Ruhigstellung, die Einsteifung, bedacht werden. Die Ruhigstellung erfolgt in Intrinsicplus-Position, um die wesentlichen Seitenbandanteile der Fingergelenke ausgespannt zu halten und damit einer Einsteifung vorzubeugen.

Knöcherne Verletzungen der Phalangen sollten möglichst innerhalb der 1 . Woche nach dem Trauma definitiv versorgt werden.

Auch wenn eine konservative Behandlung möglich wäre, macht es u.U. Sinn, operativ vorzugehen, nämlich dann, wenn dadurch eine umgehende Bewe-

OP-JOURNAL 2015; 31: 68-71

(c) Georg Thieme Verlag KG Stuttgart · New York DOI http://dx.doi.org/10.1055/s-0035-1546263 gungsübung ermöglicht wird. Dies ist bei exakt ausgeführten Schrauben- und Plattenosteosynthesen möglich, bei über den Knochen hinausragenden K-DrahtRetentionen meist nicht.

Eine klare Operationsindikation besteht beim Drehfehler des Fingers, bei der dislozierten und bei der instabilen Fraktur. Offene Phalanxfrakturen sind häufig und sollten ebenfalls operativ versorgt werden. Der versierte Handchirurg hat dabei nicht nur den Knochen und die Gelenke im Blick, sondern ebenso die Weichteile. Grundsätzlich gilt, dass Sehnen- und Bandverletzungen, Verletzungen der Gefäß-Nerven-Bündel und Weichteildefekte primär mitversorgt werden sollten.

Eine klare Operationsindikation besteht beim Drehfehler des Fingers.

\section{Endphalanxfrakturen}

Ätiologie

Endphalanxfrakturen sind meistens Folge von Quetschtraumen oder axialen Stauchungstraumata z. B. im Ballsport.

\section{Klassifikation}

Man unterscheidet Nagelkranzfrakturen, Schaftfrakturen und Basisfrakturen mit oder ohne Gelenkbeteiligung. Eine Sonderform ist der knöcherne Streck- oder Beugesehnenausriss.

\section{Nagelkranzfrakturen}

Nagelkranzfrakturen werden i.d.R. konservativ in einer Endgelenklagerungsschiene für einige Wochen ausbehandelt. Die dabei fast immer dislozierten kleinen Fragmente müssen nicht reponiert werden. Man sollte primär auf Weichteilverletzungen achten und ggf. z.B. ein subunguales Hämatom entlasten. Die handelsüblichen Stack-Schienen passen oft nicht. Individuell angepasste Schienen aus thermoplastisch verformbarem Kunststoff sind meist die bessere Wahl.

\section{Schaftfrakturen}

Schaftfrakturen können, falls es sich um stabile, nicht dislozierte Frakturen handelt, konservativ in einer Endgelenklagerungsschiene behandelt werden. Falls es sich um eine instabile Fraktur handelt, wird eine Retention unter Verwendung von 2 K-Drähten der Stärke 0,6-0,8 mm durchgeführt. Dickere Drähte und mehrere Bohrversuche sowie $\mathrm{zu}$ schnelles Bohren (Hitzeentwicklung der den Knochen verdrängenden K-Drähte) schädigen den Gelenkknorpel und den Knochen unnötigerweise. Die Drähte werden unter Röntgenkontrolle von distal nach proximal, parallel eingebracht und 4-6 Wochen belassen.

\section{Knöcherner Strecksehnenausriss}

Beim knöchernen Strecksehnenausriss mit Gelenkbeteiligung gilt, dass eine Operationsindikation besteht, wenn das Fragment mehr als ein Drittel der Gelenkfläche einnimmt. Die Fragmente können sowohl offen als auch geschlossen reponiert werden. Bewährt hat sich 


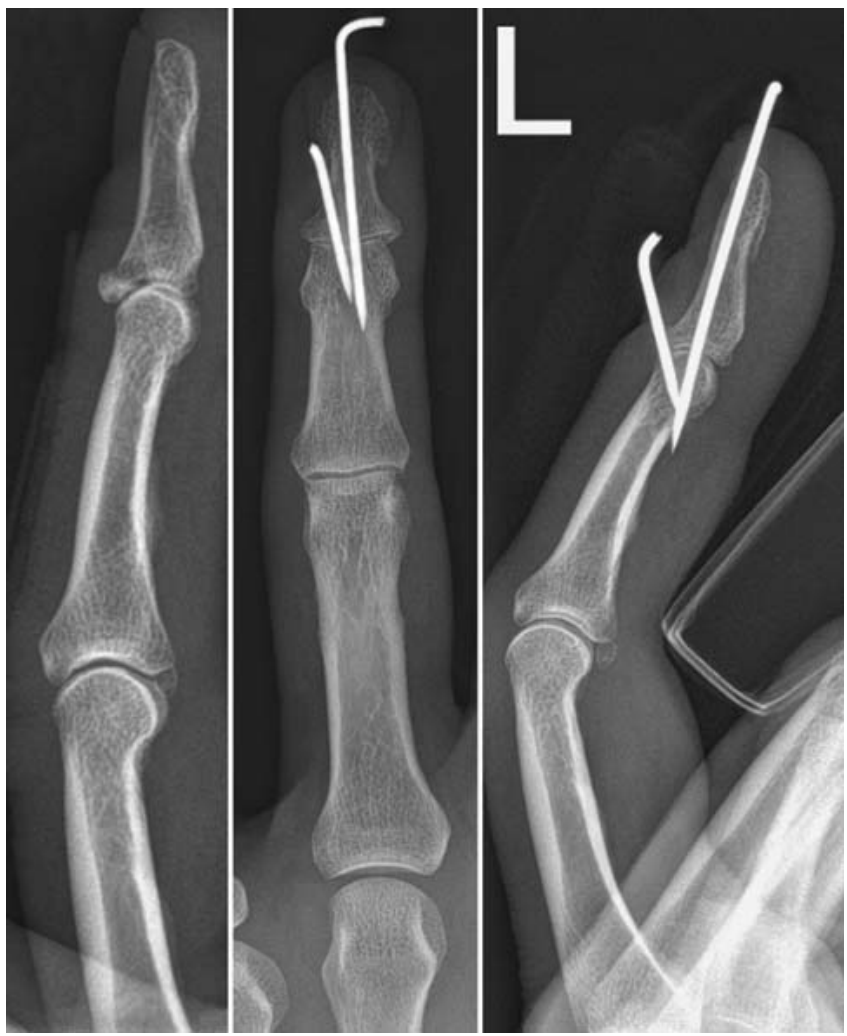

Abb. 140 Jahre, knöcherner Strecksehnenausriss. Geschlossene Reposition und Transfixierung anch Ishiguro. die geschlossene Technik nach Ishiguro, bei der zunächst ein K-Draht im Sinne eines Extensionsblocks eingebracht wird und dann das Fragment über einen von distal eingebrachten Draht in einer Extensionsbewegung dagegen reponiert wird [1]. Der von distal eingebrachte Draht wird dann nach proximal vorgebohrt. Er dient zur Retention der Fragmente und transfixiert das Gelenk bis zur Abheilung (4-5 Wochen). Manchmal ist ein weiterer Draht im Sinne eines „Joysticks“ notwendig, um ein Verdrehen des Fragments beim Repositionsmanöver zu verhindern [6]. Die Drahtstärke sollte höchstens $1,0 \mathrm{~mm}$ betragen (Abb. 1).

\section{Knöcherner Beugesehnenausriss}

Der dislozierte knöcherne Beugesehnenausriss stellt immer eine Operationsindikation dar. Das Fragment wird offen reponiert und retiniert, um eine Beugeinsuffizienz zu verhindern. Falls die Größe des Fragments eine Schraubenosteosynthese nicht zulässt, hat sich an der Endphalanx die transossäre Refixierung von palmar nach dorsal mittels einer Naht bewährt. Die Naht wird über dem Nagel verknotet.

\section{Mittel- und Grundphalanxfrakturen}

\section{Ätiologie}

Mittel- und Grundphalanxverletzungen sind meistens Folge von direkten Stauchungs-, Verdreh- oder Quetschungstraumata. Nicht selten ist auch eine Phalanxfraktur, welche aus einer Luxation des Mittelgelenks resultiert.

\section{Klassifikation}

Es werden Kopf-, Schaft- und Basisfrakturen unterschieden. Kopf- und Basisfrakturen können sowohl extra- als auch intraartikulär vorliegen (Abb. 2). Weiterhin kann ein knöcherner Ausriss des Strecksehnenmittelzügels oder der eigentlichen Seitenbänder vorliegen. Klinisch sollte v.a. bei den Schaftfrakturen auf einen Drehfehler immer im Vergleich zur gesunden Hand geachtet werden.

\section{Diagnostik}

Zur Bildgebung reichen i.d.R. eine native dorsopalmare und eine streng seitliche Zielröntgenaufnahme des betroffenen Strahles aus. Wichtig ist es, das Grundund Mittelgelenk dabei exakt abzubilden, um diese bez. der Stellung und Frakturbeteiligung korrekt beurteilen zu können. Die Kondylen sollten dabei kongruent abgebildet werden. Ein Röntgenbild der ganzen Hand hingegen ist zu ungenau und lässt eine differenzierte Beurteilung v.a. der Fingergelenke nicht zu. Bei komplexen Gelenkfrakturen kann u.U. ein exakt durchgeführtes $\mathrm{CT}$ bei der Beurteilung und OP-Planung helfen.

Zur Bildgebung reichen i.d. R. eine native dorsopalmare und eine streng seitliche Zielröntgenaufnahme des betroffenen Strahles aus.

\section{Therapie}

Bei der Versorgung gelten o.g. Prinzipien. Bei intraartikulären Frakturen sollte die betroffene Gelenkfläche möglichst exakt reponiert werden, um die sekundäre Arthrosegefahr zu minimieren.

\section{Schaftfrakturen}

Schaftfrakturen sind häufig lang und spiralförmig verlaufend und können oft auch aufgrund dieser langen Frakturstrecke mittels einzelner Schrauben stabil gestellt werden. Diese Versorgung kann gegenüber der Plattenosteosynthese oft weniger traumatisch durchgeführt werden. Die exakte Länge der Mittelund Grundphalanx ist von Bedeutung, da bei Verkürzung v.a. die Strecksehnenfunktion zur Insuffizienz neigt. Falls eine Plattenosteosynthese unumgänglich ist, führt diese über einen Mitt-Seit-Zugang eingebracht meist zu weniger Strecksehnenverklebungen und damit $\mathrm{zu}$ einer besseren und früheren Beweglichkeit im Vergleich zum dorsalen Zugang mit einer Plattenosteosynthese unter dem Strecksehnenapparat (Abb. 3).

\section{Basisgelenkfraktur}

Bei der Basisgelenktrümmerfraktur der Mittelphalanx (Pilonfraktur) kann ein Bewegungsfixateur nach Suzuki indiziert sein [4,5]. Die Extensionskomponente des Fixateurs nutzt den Ligamentotaxiseffekt aus und bewirkt dadurch eine Reposition, oft auch kleinerer Fragmente, die nicht mehr durch eine Osteosynthese reponiert und fixiert werden können. Gleichzeitig kann mit aktiven Extensions-/Flexionsübungen sofort postoperativ begonnen werden. Der Bewegungsfixateur kann mittels KDrähten und Gummizügeln intraoperativ relativ einfach selbst angefertigt werden. Er sollte nach ca. 4-5 Wochen, je nach klinischem und radiologischem Befund, wieder entfernt werden. Eingestauchte Gelenkanteile können mittels eines an der Spitze umgebogenen K- 

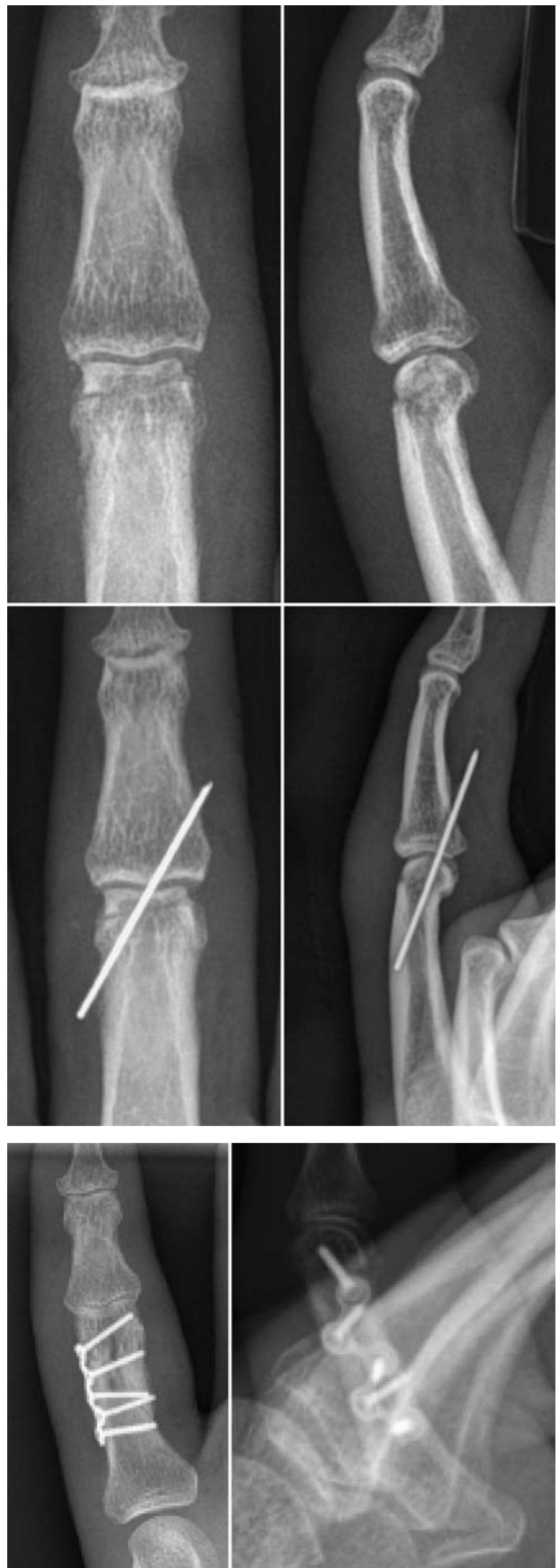

Abb. 242 Jahre, offene Kondylenfraktur, undisloziert, instabil. Teilverletzung des Strecksehnenmittelzügels. Naht der Sehne und Transfixation palmar der Fraktur um diese nicht zu dislozieren (K-Draht $0,8 \mathrm{~mm})$ für 3 Wochen.

Abb. 3 Grundgliedschaftfraktur, mehrfragmentär. Laterale Plattenosteosynthese.
Drahtes der Stärke 1,0 mm, von distal intramedullär über ein kleines Knochenfenster eingebracht, in die korrekte Position gestößelt werden [3].

Alternativ ist primär oder sekundär der Hemihamatumtransfer möglich. Dabei wird der distale, gelenktragende Anteil des Os hamatum als Teilgelenkflächenersatz für den palmaren distalen Anteil des Mittelgelenks verwendet. Dieser wird von palmar eingebracht und mittels einer Schraubenosteosynthese stabilisiert [2].

In Fehlstellung stehende Gelenkfrakturen des DIP (distales Interphalangealgelenk) und PIP (proximales Interphalangealgelenk) können auch noch nach mehr als 4 Wochen erfolgreich, im Sinne einer intraartikulären Frühkorrektur, revidiert werden. Ohne Korrektur resultiert fast immer eine posttraumatische Arthrose mit zunehmender, oft schmerzhafter Einsteifung.

\section{Frakturen im Wachstumsalter}

Bei dislozierten Phalanxfrakturen im Wachstumsalter mit Beteiligung der Wachstumsfugen sollte eine sorgsame, exakte und zügige Reposition erfolgen. Zur Fixierung werden möglichst dünnkalibrige K-Drähte $(0,6-0,8 \mathrm{~mm})$ verwendet, zum einen, um die Wachstumsfugen so wenig wie möglich zu traumatisieren, zum anderen, um eine rasche und unproblematische Entfernung derselben gewährleisten zu können (Abb.4). Noch mehr als bei Erwachsenen gilt, dass jegliche Hitzeentwicklung durch zu schnelles Vorbohren des Drahtes zu unterlassen sind, sowie mehrfache Bohrversuche einen vermeidbaren Flurschaden hinterlassen. Eine oszilierende Bohrtechnik ist zur Vermeidung sinnvoll. Die Drahtentfernung kann meist bereits nach 3-4 Wochen erfolgen.

Zu schnelles Vorbohren des Drahtes sowie mehrfache Bohrversuche verursachen Knochen- und Weichteilnekrosen.

\section{Ausrisse des Strecksehnenmittelzügels}

Nicht dislozierte knöcherne Ausrisse des Strecksehnenmittelzügels werden in einer Mittelgelenklagerungsschiene in Streckstellung für 4-6 Wochen versorgt. Durch die dabei mögliche aktive Bewegung des Endgelenks werden die Seitenzügel und der Mittelzügel nach distal verlagert. Die dadurch entstehende biologische Zuggurtung wirkt der Dislokation entgegen [7]. 


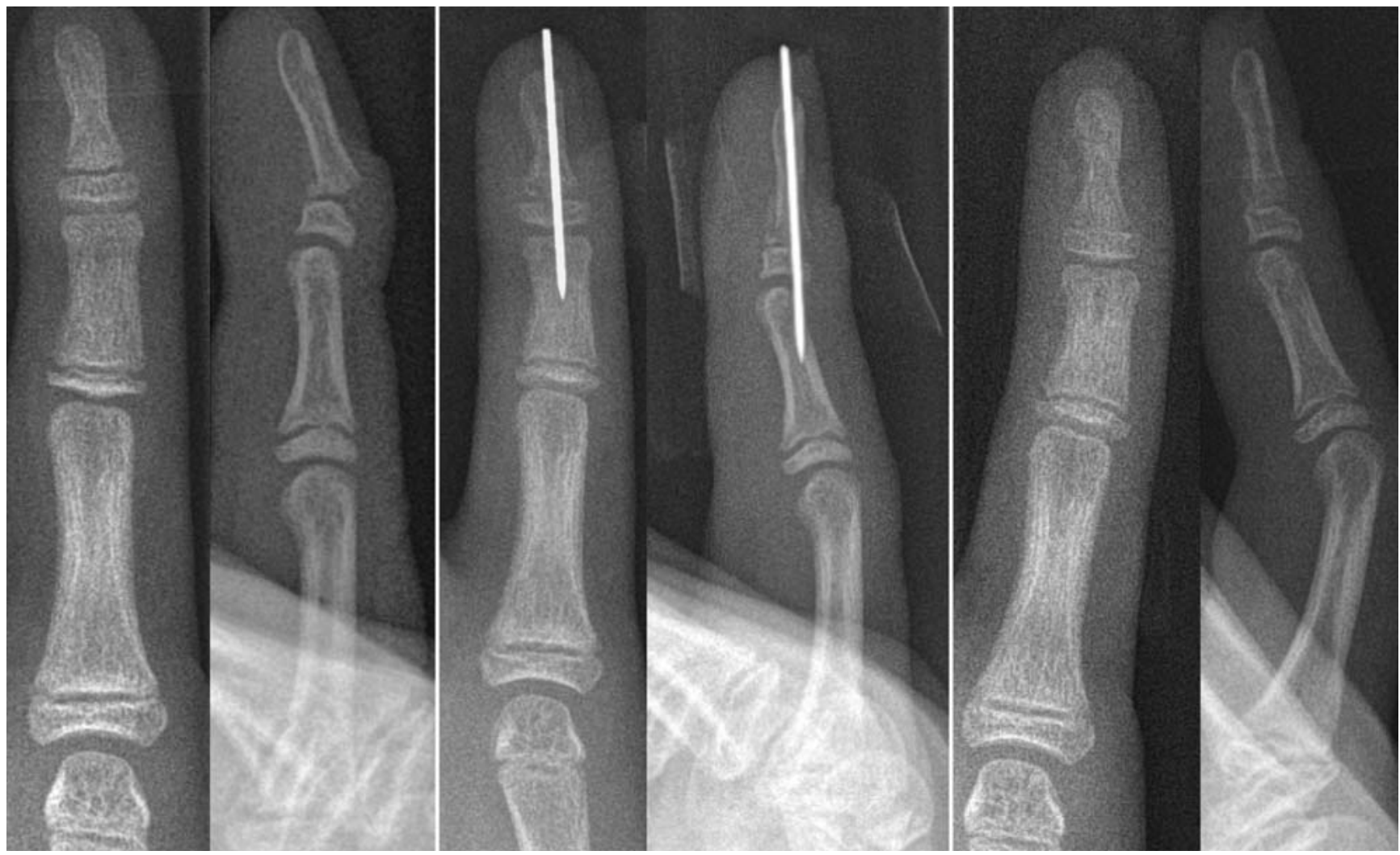

Abb. 415 Jahre, offene Epiphysiolyse Endphalanx, Nagelluxation, Nagelbett verletzt. Reposition und Transfixation (K-Draht 0,8 mm), Nagelbettnaht, Nagelreposition (Metallentfernung nach 3 Wochen, keine weitere Ruhigstellung).

\section{Daumenfrakturen}

Ätiologisch und klassifizierend gilt die gleiche Einteilung wie bei den 3-gliedrigen Fingern. Allerdings ist die Beweglichkeit der Interphalangealgelenke des Daumens nicht in dem Maße entscheidend für die Funktion, wie es bei den 3gliedrigen Fingergelenken der Fall ist. Für die Daumenfunktion ist v.a. die Stabilität von Bedeutung, während für die 3-gliedrigen Finger eher die intakte Beweglichkeit wichtig ist. Am Daumen haben über die beschriebenen Frakturen hinaus knöcherne Seitenbandausrisse eine Bedeutung. Dislozierte Fragmente sollten offen reponiert und mittels einer Schraubenosteosynthese $(1,0-1,5 \mathrm{~mm})$ retiniert werden. Nicht dislozierte Fragmente können konservativ zur stabilen Ausheilung gebracht werden.

\section{Komplikationen}

Die Einsteifung der Finger ist bei Weitem die häufigste Komplikation im Rahmen der Frakturversorgung. Eine möglichst wenig traumatische Technik und die konsequente und kompetent geführte Frühmobilisation sind das Geheimnis, um eine gute Beweglichkeit zu erhalten. Pseudarthrosen sind selten. Die Infektionsrate ist selbst bei über Hautniveau stehenden K-Drähten und bestehenden Komorbiditäten klein [9]. Zur Kälteintoleranz kommt es jedoch, zumindest passager, häufig [8].

Die Einsteifung der Finger ist bei Weitem die häufigste Komplikation im Rahmen der Frakturversorgung. Eine möglichst wenig traumatische Technik und die konsequente und kompetent geführte Frühmobilisation sind das Geheimnis, um eine gute Beweglichkeit zu erhalten.

\section{Literatur}

${ }^{1}$ Ishiguro T, Yabe Y, Itoh Y et al. Extension block with Kirschner wire for fracture dislocation of the distal interphalangeal joint. Tech Hand Up Extrem Surg 1997; 1: 95e102

${ }^{2}$ Rafael MM, Williams MD, Thomas $R$ et al. Treatment of unstable dorsal proximal interphalangeal fracture/dislocations using a hemi-hamate autograft. J Hand Surg 2003; 28 A: 856-865

${ }^{3}$ Hintringer W, Ender HG. Perkutane Versorgung von intraartikulären Frakturen der Fingermittelglieder. Handchir Mikrochir Plast Chir 1986; 18: 356-362

${ }^{4}$ Suzuki Y, Matsunaga T, Sato $S$ et al. The pins and rubbers traction system for treatment of comminuted intraarticular fractures and fracture-dislocations in the hand. J Hand Surg Br 1994; 19: 98-107

${ }^{5}$ Stern PJ, Roman RJ, Kiefhaber TR et al. Pilon fractures of the proximal interphalangeal joint. J Hand Surg Am 1991; 16: 844-850

${ }^{6}$ Miura T. Extension block pinning using a small external fixator for mallet finger fractures. J Hand Surg Am 2013; 38: 2348-2352

7 Pillukat T, Mühldorfer-Fodor M, Schädel-Höpfner $M$ et al. Verletzungen der Mittelgelenke. Unfallchirurg 2014; 117: 315-326

${ }^{8}$ Nijhuis T, Smits E, Jaquet $J$ et al. Prevalence and severity of cold intolerance in patients after hand fracture. J Hand Surg Eur Vol 2010; 35: 306-311

${ }^{9}$ Hsu L, Schwartz E, Kalainov D et al. Complications of K-wire fixation in procedures involving the hand and wrist. J Hand Surg Am 2011; 36: 610-616

\section{Dr. med. Holger C. Erne}

Oberarzt

\section{Univ.-Prof. Dr. med. Hans-Günther}

Machens

Direktor

Klinik und Poliklinik für Plastische

Chirurgie und Handchirurgie

Klinikum rechts der Isar

Technische Universität München

Ismaninger Straße 22

81675 München

machens@lrz.tum.de 